\title{
Does wrist immobilization following open carpal tunnel release improve functional outcome? A literature review
}

\author{
S. M. Isaac • T. Okoro • I. Danial • C. Wildin
}

Published online: 11 July 2010

(C) Humana Press 2010

\begin{abstract}
Carpal Tunnel Syndrome (CTS) is a compressive neuropathy of the median nerve in the carpal tunnel. It is the most common peripheral entrapment neuropathy. The surgical management includes dividing the flexor retinaculum to decompress the median nerve. Post-operative mobilization of the wrist is controversial. Some surgeons splint the wrist for 2-4 weeks whilst others encourage early mobilization. The literature has been inconclusive as to which method is most beneficial. The purpose of this study is to review the literature regarding the effectiveness of wrist immobilization following open carpal tunnel decompression. We reviewed all published clinical trials claiming to evaluate the mobility status following open carpal tunnel release. Studies not in the English language as well as those with small number of patients $(n<30)$ were excluded. There were five studies that fulfilled the eligibility criteria and were included in this review. We conclude that there is no beneficial effect from post-operative immobilization after open carpal tunnel decompression when compared to early mobilization.
\end{abstract}

Keywords Carpal tunnel syndrome - Open release . Decompression $\cdot$ Immobilization $\cdot$ Splinting

\footnotetext{
S. M. Isaac $(\varangle) \cdot C$. Wildin

Trauma and Orthopaedics, University Hospitals of Leicester, Glenfield Hospital, Groby Road, Leicester LE3 9QP, UK e-mail: sherifisaac@hotmail.com

T. Okoro

Trauma and Orthopaedics, Leicester Royal Infirmary, Infirmary Square, Leicester LE1 5WW, UK

I. Danial

Northampton, UK
}

\section{Introduction}

Carpal tunnel syndrome was first described by Sir James Paget in 1854 and is the most common peripheral nerve compressive neuropathy [1]. It involves compression of the median nerve in the carpal tunnel at the wrist. The majority of cases are idiopathic but there are associated risk factors such as diabetes mellitus, rheumatoid arthritis and pregnancy [2]. The typical symptoms are pain especially at night, paresthesia in the distribution of the median nerve and wasting of the thenar muscles in advanced cases [3].

The majority of patients with CTS are treated convservatively. This includes analgesia, non-steroidal anti-inflammatory drugs, splinting, activity modification, physical therapy and local steroid injection [3-7]. The benefit of splinting in the treatment of CTS is controversial. A systematic review by Gerritsen in 2002 suggested that there is little evidence to suggest that splinting is less effective than surgery in providing long-term relief from symptoms [8]. Later on, the same author published the results of a randomized controlled trial comparing splinting versus surgery in the treatment of CTS and contradictory concluded that open carpal tunnel release resulted in better outcomes than wrist splinting [9]. A recent systematic review suggested that splints are an effective method in the conservative management of CTS especially if used full-time [3]. Carpal tunnel decompression is the accepted surgical treatment when conservative measures fail [10] and open decompression under local anaesthesia remains the standard surgical technique.

Post-operative rehabilitation regimes are controversial. Splinting is common after carpal tunnel release, despite a lack of scientific evidence supporting its value [11], and some surgeons still prefer to immobilize the wrist in a splint for a period of 2-4 weeks. The theoretical 
advantages mentioned by those proponents have included patient comfort, avoidance of wound dehiscence, prevention of bowstringing of the flexor tendons, prevention of subluxation and tethering of the median nerve in the scar and improved grip strength [12-18]. There are no available valid data or study materials to support that post-operative splinting benefits these theoretical advantages. Others advocate early movement to promote longitudinal gliding of the nerve through the surgical bed [19]. In addition, it has been suggested that post-operative splinting caused a significant delay in return to work or activities of daily living [20].

This study is aimed at reviewing the available literature to identify whether splinting the wrist following carpal tunnel release is superior to early mobilization in improving functional outcome. This is the first literature review in the English literature to assess wrist splinting versus immediate mobilisation after carpal tunnel decompression.

\section{Methods and materials}

\section{Eligibility}

Table 1 illustrates the inclusion and exclusion criteria used for assessing eligibility.

\section{Study identification}

Studies were identified from computerized search of MEDLINE (1950 to date), EMBASE (1980 to date), COCHRANE database and by review of the references found in the articles thus recovered. The MEDLINE and EMBASE search was done on OvidSP (available at http://ovidsp.tx.ovid.com/ spa/ovidweb.cgi). A set was created using the terms: 'carpal tunnel syndrome'; yielded 6514 articles. A search strategy was then built by adding the terms 'immobilization', 'splinting', 'rehabilitation' and 'surgical decompression' to 'carpal tunnel syndrome'.
Data extraction

All relevant information regarding the population, intervention, post-operative rehabilitation, recovery and the development of complications.

Outcome measures

Grip and pinch strength, range of wrist and fingers' movement, visual analogue scale for pain and scar tenderness and complication rates.

\section{Results}

Four hundred and fourteen articles were thus recovered after the computerized search. These articles were then limited to randomized controlled trials (1b Level of Evidence) to yield only five documents that satisfied the inclusion criteria and actually dealt with the mobility status following open carpal tunnel release [1, 20-23].

These five articles subsequently underwent review, analysing both their methodological quality and the effects of both regimes. Table 2 provides a pertinent summary of the analysed articles.

In 1995, Bury et al. published a prospective randomized trial to determine the possible beneficial effect of postoperative splinting after open carpal tunnel release [1]. It was not clear if the authors obtained an ethical approval for the trial. The diagnosis of CTS was made clinically and confirmed by electrophysiological studies. Post-operatively, patients were randomized into two groups to receive either a bulky dressing with splint for 2 weeks or a bulky dressing only. Randomization was done by a random number generator.

Patients were reviewed once post-operatively at an average of 6 months (range, 3.8-7.8 months). This wide range in follow-up between patients, which is more than double, would have implications when assessing outcome. At follow-up, patients were evaluated for residual or
Table 1 Inclusion and exclusion criteria used for assessing eligibility

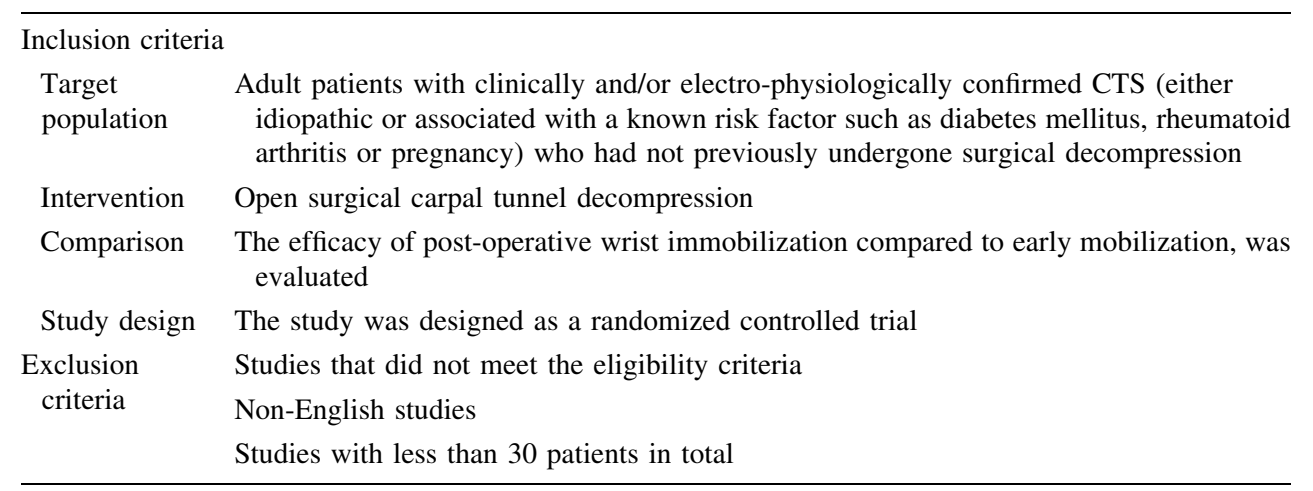




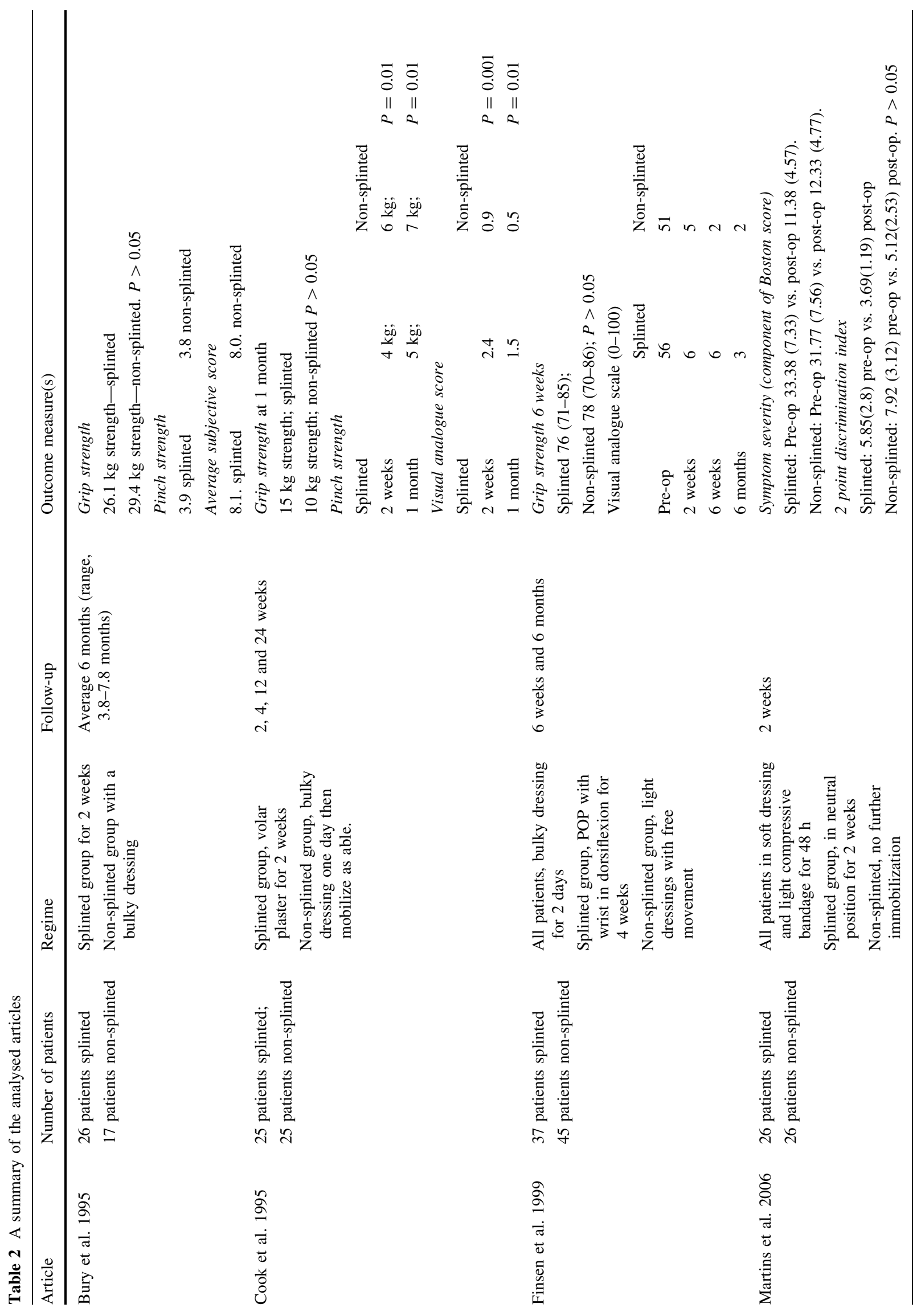




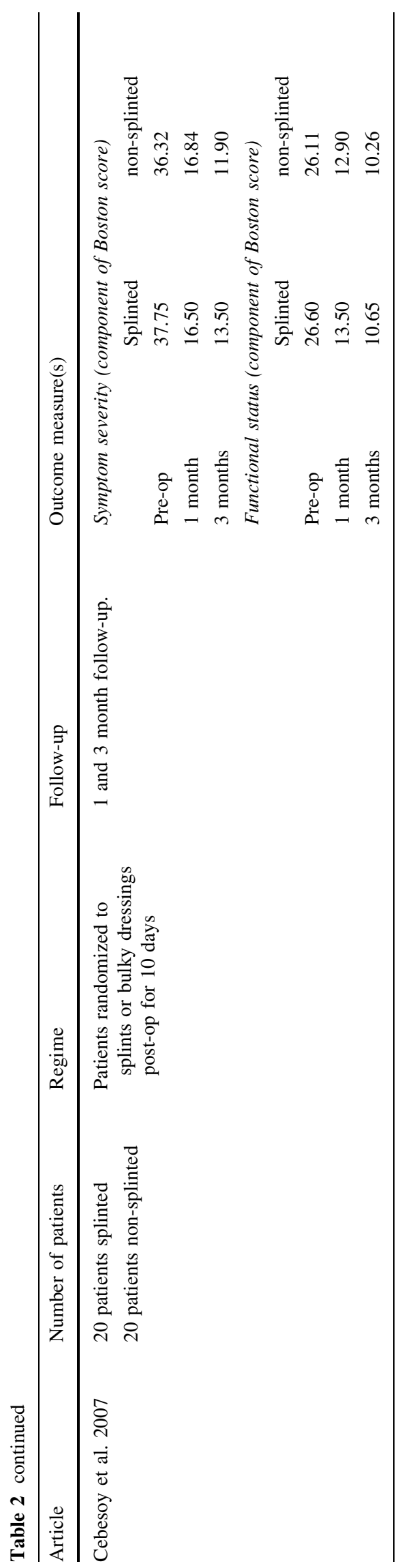

recurrent symptoms, digital and wrist range of motion, grip and pinch strength, subjective outcome and patient satisfaction score and the development of complications. This subjective outcome score that the authors used to assess outcome was not referenced.

The authors compared post-operative results with the contra-lateral side, which was affected with carpal tunnel syndrome in $80 \%$ of cases, and also with normal average grip and pinch strength tables, corrected for gender, age and hand dominance. This means that patients were not assessed longitudinally in a way that there was no preoperative assessment to be compared with the postoperative measurements. Therefore, this may have drawn inappropriate conclusions. This is clear from the results which showed that the post-operative group scores were higher in grip strength than the control group. There is a great variability in grip strength between patients and to minimize this effect of variability it is essential to use the patients as their own control to compare pre- and post-operative data in the same side. This could be one of the reasons why these scores were not statistically significant.

One of the observations of this study was that splinting had delayed return to work. There were $27 \%$ of patients in the splinted group that had not returned to work at last follow-up compared to $12 \%$ in the non-splinted group. All but one of these cases involved worker's compensation coverage and this might explain the delay in return to work. The authors then concluded that they found no benefit in outcome by using post-operative splinting.

In the same year, Cook et al. conducted a prospective randomized study of 50 patients that underwent surgery for CTS to determine the value of wrist splintage following open decompression [20]. All the patients underwent the same surgical procedure and then were randomized into two groups. The first group had a volar plaster splint for 2 weeks while the second group was allowed an unrestricted active motion from the first post-operative day. Patients were then evaluated at 2, 4, 12 and 24 weeks. The authors did not mention what method of randomization was used. The outcome measures used were return to normal activity and work, grip and pinch strength, two-point discrimination, sensibility, complications and a post-operative subjective pain score. Post-operatively, the two groups were similar except for pain score, outcome rating and grip and pinch strength that were worse in the splinted group, only in the short term. Return to normal activity and work was more rapid in the un-splinted group.

Despite the assessment was done in all patients preoperatively, there was no longitudinal comparison between pre and post-operative measurements and data were compared only between groups.

The authors then concluded that the value of splinting in prevention of complications is not valid but they admitted 
that this could be due to the small sample size. In addition, they claimed that immobilization has resulted in more pain, stiffness and delay to return to work. Surprisingly at the end of their recommendations, they suggested that if splints had to be used as a precaution against tendon bowstringing and nerve entrapment, it should be limited to 1 week despite their data results did not support this recommendation.

Finsen et al. in 1999 studied the value of wrist splinting following open decompression in 82 wrists [21]. In this study, there were no clear inclusion and exclusion criteria. The diagnosis was mostly based on history and examination but few patients had nerve conduction studies to confirm the diagnosis. Depending on history and examination to diagnose CTS is usually enough in the normal clinical sitting, but in a clinical trial which was set up to confirm or exclude a hypothesis, uniformity and certainty in diagnosis must be achieved before any conclusion can be withdrawn.

All the patients had the same surgical procedure and then were randomized to one of two post-operative regimens. The method of randomization was done by adding up the 11 digits in the patients' social security number and odd numbers constituted one group while even numbers constituted the second group. Post-operatively, one group was allowed to mobilize as pain allows and the other group had a wrist splint for 4 weeks. Patients who were operated on bilaterally had one regimen for one hand and the other regimen for the second hand. The patients were evaluated at 6 weeks and 6 months post-operatively. The evaluation included grip and pinch strength and a visual analogue pain score. This visual analogue scale subjectively measured pain level pre- and post-operatively. Patients were asked to disregard pain which had risen following surgery but only residual pain that they had had pre-operatively. This is clinically difficult to assess and in actual fact the postoperative surgical pain should have also been measured as this is the pain that wrist splinting was claimed to improve and is one of the reasons why splints have been used.

Surprisingly, this study showed that more patients in the mobilized group were sick-listed $(n=28)$ compared to the immobilized group $(n=19)$. The authors explained this to be due to the agreed time of sick leave between the surgeon and the patient.

There was no difference between the groups in regard to grip and pinch strength compared to pre-operative evaluation but the measurements were not performed by the same assessor. This somewhat, could have affected the reliability of the measurements and it seemed that the authors had intentionally done this to reduce bias. The authors concluded that splinting even for 4 weeks had no advantage on outcome following open carpal tunnel release.
Martins et al. studied the possible advantages of wrist immobilization after open carpal release in a small series of 52 patients with CTS [22]. Diagnosis was based on clinical examination and electrophysiological studies. There were specific inclusion and exclusion criteria. Patients had tried conservative management for 6 weeks before listed for surgery.

All patients underwent an open surgical decompression performed by one surgeon. After an initial period of immobilization for $48 \mathrm{~h}$, patients were randomized to either splinting for 2 weeks or to mobilize freely. The randomization method was not stated. Outcome measures used were two-point discrimination test and two questionnaires of symptoms severity and intensity. The evaluation was performed pre- and 2 weeks post-operatively by the same assessor. The two questionnaires were the Severity Symptom Score or Boston questionnaire which is a validated questionnaire [24] and the Symptom Intensity Scale that was not referenced and was not clear if this questionnaire has been previously validated. Grip and pinch strength as well as range of motion were not evaluated. Pre- and post-operative data were then compared for each patient. There was no statistically significant difference between the two groups.

The last article by Cebesoy et al. published in 2007, was undertaken to compare the clinical effectiveness and costs of post-operative splints versus early rehabilitation [23]. A total of 40 patients were recruited in this study. Diagnostic criteria consisted of only history and clinical examination. This again as stated before may not be enough to reach an appropriate conclusion. There were definitive inclusion and exclusion criteria. Patients underwent the same surgical procedure. Randomization was designed according to chart numbers, so that even numbers were assigned to wear a splint for 10 days while odd numbers were given a bulky bandage and were allowed mobilization. Post-operatively, patients were assessed at 1 and 3 months and only included the Questionnaire of Levine [24]. The authors found no statistically significant difference between the two groups. The authors also stated that bulky bandages were nine times cheaper than splints.

\section{Discussion}

Open carpal tunnel decompression is one of the most commonly used procedures for peripheral entrapment mono-neuropathy after failed conservative measures. Hand weakness after open carpal tunnel release has been an exciting point of discussion and its explanation remains a question without a clear answer. Pain, atrophy, altered sensation, widening of the carpal arch and bowstringing of the flexor tendons have all been implicated as possible causes [25, 26]. 
The flexor retinaculum serves as a pulley restraining the flexor tendons when flexing the wrist [20]. Theoretically, division of this restrain may allow bowstringing of the tendons, entrapment of the median nerve in the scar, wound dehiscence and pain. Therefore, post-operative splinting has been advocated [12-18] and a survey conducted in 1987 showed that most American Hand Surgeons splint the wrist post-operatively for 1-4 weeks [5]. However, published studies have not supported these theories. Nathan et al. allowed early mobilization in 216 decompressions and reported no such problems [19]. Earlier in 1976, Das et al. reported no volar prolapse of the median nerve or flexor tendons in 170 cases [27].

From the presented data, all studies seem to come to the same conclusion that splinting is of no value in the postoperative rehabilitation following carpal tunnel release. The complications of tendon bowstringing or adherence of the nerve to the scar that splints were claimed to avoid, seem to be very rare and were not of any concern at least in the five articles that were analysed in this review. Nevertheless, the follow-up in most of these studies was short but it seems reasonable to discharge patients when full normal activity was regained.

This review shows some evidence that using splints post carpal tunnel decompression does not improve outcome and in some cases have caused more problems and delay in return to work and normal activities. Although, all reviewed articles showed distinct differences in design such as splinting period, follow-up time and outcome measures, based on these reviewed studies there seems to be no evidence to support the use of splints in the postoperative period. The use of splints might be beneficial in certain circumstances such as revision surgery when longer incision is used or in rheumatoid patients undergoing simultaneous synovectomy but this theory is to be proven.

This study did not include trials in languages other than English and this could be one of its limitations, and, therefore, more evidence is needed before appropriate conclusions can be drawn. Clearly, this review confirms that the available data regarding wrist immobilization following surgery is still unsatisfactory and none of the five studies could show an apparent advantage of wrist splinting following surgical release. Therefore, a rigorous randomized multi-centre trial of adequate power is needed to determine the efficacy of splint immobilization following surgical carpal tunnel decompression.

\section{References}

1. Bury TF, Akelman E, Weiss APC. Prospective randomised trial of splinting after carpal tunnel release. Ann Plast Surg. 1995; 35(1):19-22.
2. Gelberman RH, Eaton R, Urbaniak JR. Peripheral nerve compression. J Bone Joint Surg Am. 1993;75:1854-78.

3. Piazzini DB, Aprile I, Ferrara PE, et al. A systematic review of conservative treatment of carpal tunnel syndrome. Clin Rehabil. 2007;21:299-314.

4. Gelberman RH, Aronson D, Weisman MH. Carpal Tunnel Syndrome-results of a prospective trial of steroid injection and splinting. J Bone Joint Surg. 1980;62-A:1181-4.

5. Duncan KH, Lewis RC, Foreman KA, Nordyke M. Treatment of carpal tunnel syndrome by members of the American Society for Surgery of the Hand: results of a questionnaire. J Hand Surg. 1987;12-A:384-91.

6. Quin CE. Carpal tunnel syndrome: treatment by splinting. Ann Phys Med. 1961;6:72-5.

7. Kaplan SJ, Glickel SZ, Eaton RG. Predictive factors in the nonsurgical treatment of carpal tunnel syndrome. J Hand Surg. 1990; 15-B:106-8.

8. Gerritsen AA, de Krom MC, Struijs MA, Scholten RJ, de Vet HC, Bouter LM. Conservative treatment options for carpal tunnel syndrome: a systematic review of randomised controlled trials. J Neurol. 2002;249(3):272-80.

9. Gerritsen AA, de Vet HC, Scholten RJ, Bertelsmann FW, de Krom MC, Bouter LM. Splinting vs surgery in the treatment of carpal tunnel syndrome: a randomized controlled trial. JAMA. 2002;288(10):1245-51.

10. Eversmann WW. Entrapment and compression neuropathies. In: Green DP, editor. Operative hand surgery. 3rd ed. New York: Churchill Livingstone; 1993. p. 1346-63.

11. Henry SL, Hubbard BA, Concannon MJ. Splinting after carpal tunnel release: current practice, scientific evidence, and trends. Plast Reconstr Surg. 2008;122(4):1095-9.

12. MacDonald RI, Lichtman DM, Hanlon JJ, Wilson JN. Complications of surgical release for carpal tunnel syndrome. J Hand Surg. 1978;3:70-6.

13. Inglis AE. Two unusual operative complications in carpal tunnel syndrome. A report of two cases. J Bone Joint Surg. 1980;62A:1208-9.

14. Gelberman RH, Pfeffer GB, Galbraith RT, Szabo RM, Rydevik B, Dimick M. Results of treatment of severe carpal tunnel syndrome without internal neurolysis of the median nerve. J Bone Joint Surg. 1987;69-A:896-903.

15. Brown RA, Gelberman RH, Seiler JG, et al. Carpal tunnel release: A prospective randomised assessment of open and endoscopic methods. J Bone Joint Surg. 1993;75-A:1265-75.

16. Omer GE. Median nerve compression at the wrist. Hand Clin. 1992;8:317-24.

17. Weirich SD, Gelberman RH. Changing concepts in the diagnosis and treatment of carpal tunnel syndrome. Current Orthop. 1993;7: 218-25.

18. Jessurun W, Hillen B, Huffstadt AJC. Carpal tunnel syndrome: postoperative care. Hand Chir. 1988;20:39-40.

19. Nathan PA, Meadows KD, Keniston RC. Rehabilitation of carpal tunnel surgery patients using a short surgical incision and an early program of physical therapy. J Hand Surg. 1993;1-A:1044-50.

20. Cook AC, Szabo RM, Birkholz SW, King EF. Early mobilization following carpal tunnel release. A prospective randomized study. J Hand Surg [Br]. 1995;20(2):228-30.

21. Finsen V, Andersen K, Russwurm H. No advantage from splinting the wrist after open carpal tunnel release. A randomized study of 82 wrists. Acta Orthop Scand. 1999;70(3):288-92.

22. Martins RS, Siqueira MG, Simplicio H. Wrist immobilization after carpal tunnel release: a prospective study. Arq Neuropsiquiatr. 2006;64(3A):596-9.

23. Cebesoy O, Kose KC, Kuru I, Altinel L, Gul R, Demirtas M. Use of a splint following open carpal tunnel release: a comparative study. Adv Ther. 2007;24(3):478-84. 
24. Levine DW, Simmons BP, Koris MJ, et al. A self-administered questionnaire for the assessment of severity of symptoms and functional status in carpal tunnel syndrome. J Bone Joint Surg. 1993;75-A:1585-92.

25. Gartsman GM, Kovach JC, Crouch CC, et al. Carpal arch alteration after carpal tunnel release. J Hand Surg. 1986;11-A:372-4.
26. Young VL, Logan SE, Fernando B, et al. Grip strength before and after carpal tunnel decompression. South Med J. 1992;85: 897-900.

27. Das SK, Brown HG. In search of complications in carpal tunnel decompression. The Hand. 1976;8(3):243-9. 